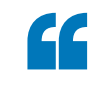

the ALS [CSF] samples were found to have an inhibitory effect on $\mathrm{SHH}$ signalling

\title{
CSF suppresses sonic hedgehog in amyotrophic lateral sclerosis
}

The cerebrospinal fluid (CSF) of patients with amyotrophic lateral sclerosis (ALS) contains factors that inhibit sonic hedgehog $(\mathrm{SHH})$ signalling, new research indicates. The findings build on previous evidence from in vitro and in vivo models that $\mathrm{SHH}$ signalling is compromised in ALS.

"Familial and sporadic forms of ALS lead to a common clinical phenotype in spite of genetically diverse mutations or no discernible

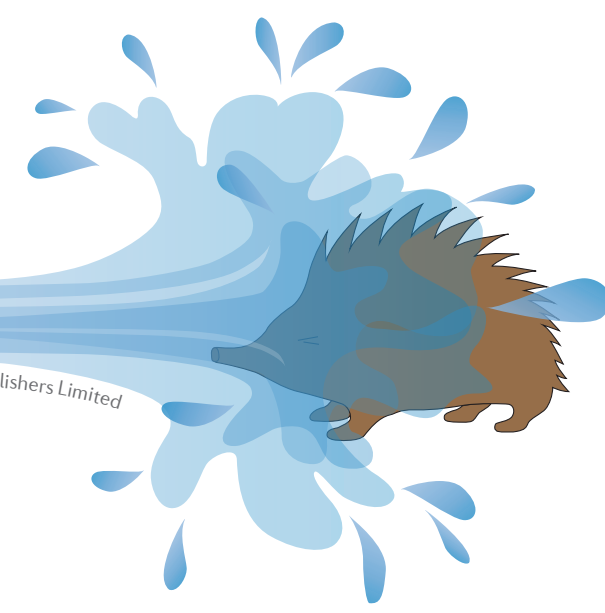

mutation at all," explains John Turnbull from McMaster University, Hamilton, Canada, who led the new study. "One factor of potential interest to a common terminal pathway is $\mathrm{SHH}$, a developmental morphogen that has multiple persisting actions, possibly including the maintenance of motor neurons in the differentiated state."

The study included nine patients with ALS, 12 controls with other neurological conditions, and 13 non-neurological controls. The researchers used ELISA to measure the levels of SHH protein in CSF samples from these individuals. In addition, they exposed GLI reporter cell lines to the CSF samples. GLI proteins are downstream transducers of $\mathrm{SHH}$ signalling, and their expression can serve as a read-out of $\mathrm{SHH}$ pathway activity.

Levels of SHH protein were comparable in CSF samples from patients with ALS and controls. However, the ALS samples were found to have an inhibitory effect on $\mathrm{SHH}$ signalling in the reporter cell lines.

"The biological activity of $\mathrm{SHH}$ was reduced in ALS CSF samples, due to an inhibitor acting below the level of the smoothened complex," reports Turnbull. "One potential culprit is tumour necrosis factor, which was increased in ALS CSF compared with controls, correlated negatively with ALS disease duration, and inhibited the biological activity of SHH in vitro."

The new findings provide the first direct evidence of impaired $\mathrm{SHH}$ signalling in the CSF of patients with ALS. "A greater understanding of the common terminal pathway for motor neuronal dysfunction in ALS might lead to successful interventions, irrespective of more-proximate causes," concludes Turnbull.

Heather Wood

ORIGINAL ARTICLE Drannick, A. et al. Cerebrospinal fluid from patients with amyotrophic lateral sclerosis inhibits sonic hedgehog function. PLoS ONE 12, e0171668 (2017) 